\title{
Bodies of constant width in arbitrary dimensions
}

\author{
Thomas Lachand-Robert \& Édouard Oudet* \\ v0.31 - 28th July 2005
}

\section{Introduction}

A body (that is, a compact connected subset $K$ of $\mathbb{R}^{n}$ ) is said to be of constant width $\alpha$ if its projection on any straight line is a segment of length $\alpha \in \mathbb{R}_{+}$, the same value for all lines. This can also be expressed by saying that the width map

$$
w_{K}: \nu \in \mathbb{S}^{n-1} \longmapsto \max _{x \in K} \nu \cdot x-\min _{x \in K} \nu \cdot x
$$

has constant value $\alpha$. This is also equivalent to the geometrical fact that two parallel support hyperplanes on $K$ are always separated by a distance $\alpha$, independent of their direction. Note that the width of a body $K$ and of its convex hull are the same. So, as many authors do, we will focus here on convex bodies of constant width.

Obvious bodies of constant width are the balls; but they are many others. These bodies, also called orbiforms in dimension two, or spheroforms in dimension three (as in [1]), have many interesting properties and applications. Orbiforms in particular have been studied a lot during the nineteenth century and later, particularly by Frank Reuleaux, whose name is now attached to those orbiforms you get by intesecting a finite number of disks of equal radii $\alpha$,

\footnotetext{
*Laboratoire de mathématiques, Université de Savoie, Campus scientifique, 73376 Le Bourget-du-lac, France. Thomas.Lachand-Robert@univ-savoie.fr, Edouard.Oudet@univ-savoie.fr http://www.lama.univ-savoie.fr/ lachand, http://www.lama.univ-savoie.fr/ oudet, http://www. lama.univ-savoie.fr/ bayen.
} 
whose center are vertices of a regular polygon of diameter $\alpha$. In particular the Reuleaux triangle is the intersection of three discs of radius $\alpha$, centered on vertices of an equilateral triangle with side $\alpha$.

So let us define formally the following class:

$$
\mathcal{W}_{\alpha}:=\left\{K \subset \mathbb{R}^{n} ; K \text { compact convex and } \forall \nu \in \mathbb{S}^{n-1}, w_{K}(\nu)=\alpha\right\} \text {. }
$$

The mere existence of non trivial three-dimensional bodies of constant width is not so easy to establish. In particular, no finite intersection of balls has constant width (except balls themselves), a striking difference with the two-dimensional case.

A simple construction is to consider a two dimensional body of constant width having an axis of symmetry (like the Reuleaux triangle for instance): the corresponding body of revolution obtained by rotation around this axis is a spheroform. F. Meissner proved [5] that the rotated Reuleaux triangle has the smaller volume among bodies of revolution in $\mathcal{W}_{\alpha}$.

Later on he was able to construct another spheroform (usually called "Meissner's tetrahedron") which does not have the symmetry of revolution. The volume of this body is smaller than any other known of constant width. We describe this body in more details later on in this paper. Let us just say for the moment that it looks like an intersection of four balls centered on the vertices of a regular tetrahedron, but some of the edges are smoothed; in particular, it doesn't have all the symmetries of a regular tetrahedron.

\section{Constant width bodies}

We recall here the main properties of bodies of constant width for convenience. Most of these are easy to prove, so we give only insight of the proofs. Details can be found in [1]. Unless otherwise stated, all bodies considered here are convex.

Let us first recall the definition of the support function for a body $K \in \mathbb{R}^{n}$ : it is the map $h_{K}: \mathbb{S}^{n-1} \rightarrow \mathbb{R}$ defined by $h_{K}(\nu):=\max _{x \in K} x \cdot \nu$. It is related to the width function by the identity:

$$
\forall \nu \in \mathbb{S}^{n-1}, \quad w_{K}(\nu)=h_{K}(\nu)+h_{K}(-\nu) .
$$

Given two bodies $K$ and $L$, their Minkowski sum is $K+L:=\{x+y$; $x \in K, y \in L\}$; more generally, we define for any $\lambda, \mu \in \mathbb{R}$, the Minkowski combination

$$
\lambda K+\mu L:=\{\lambda x+\mu y ; x \in K, y \in L\} .
$$


It follows easily from the definitions that, for $\lambda \geq 0$ and $\mu \geq 0$, we have $h_{\lambda K+\mu L}=\lambda h_{K}+\mu h_{L}$, and then $w_{\lambda K+\mu L}=\lambda w_{K}+\mu w_{L}$ as well. We also have $h_{-K}(\nu)=h_{K}(-\nu)$ for all $\nu$, so $w_{-K}=w_{K}$. We deduce generally that

$$
\forall \lambda, \mu \in \mathbb{R}, \quad w_{\lambda K+\mu L}=|\lambda| w_{K}+|\mu| w_{L} .
$$

As a consequence, the fact that $K$ has constant width can easily be expressed with a Minkowski difference, namely

$$
K-K=\alpha B_{1}
$$

where $B_{1}$ is the unit ball of $\mathbb{R}^{n}$. We see also that if $K$ has constant width $\alpha$, then $K+\beta B_{1}$ has constant width $\alpha+\beta$ for any $\beta \geq 0$.

A simple consequence of this property is that no body of constant width has a center of symmetry, unless it is a ball. Indeed if $-K$ is (a translate of) $K,(5)$ proves that $K$ is a ball of radius $\alpha / 2$. This explains why it is not possible to find an orbiform based on a square, or any even-sided polygon, as the Reuleaux polygons are based on odd-sided polygons. Similarly in dimension 3, there is no spheroforms having the same group of symmetries than the cube, octahedron, dodecahedron or icosahedron, except the ball. On the other hand there are some bodies of constant width whose group of symmetries is the same than the tetrahedron's. In order to construct one, it suffices to start from one of the Meissner's bodies $K_{1}$, that have all the required symmetries except one. So let $K_{2}$ be the symmetrical of $K_{1}$ with respect to the missing plane of symmetry. Now $K:=\frac{1}{2}\left(K_{1}+K_{2}\right)$ has constant width $\alpha$, and has all the symmetries of the tetrahedron.

For a convex body $K$, we say that a hyperplane $H$ is a hyperplane of support for $K$ at $x$, if $x \in K \cap H$ and $K$ is included in one of the half-spaces limited by $H$. If $\nu \in \mathbb{S}^{n-1}$ is a normal vector to $H$, pointing outside the half space containing $K$, we say that $\nu$ is an outward support vector at $x$. Obviously if $K$ is smooth (that is, has a differentiable boundary), then $\nu$ is just the outward unit normal at $x$. In this particular case, there is a map $x \mapsto \nu$ which is usually called the Gauss map.

Note that a body of constant width is not always smooth, as the Reuleaux triangle shows. It turns out that for our purpose, we are more interested in the reverse Gauss map: for a strictly convex body $K$, and for any given $\nu \in \mathbb{S}^{n-1}$, the linear map $x \in K \mapsto x \cdot \nu$ attains its maximum at a unique point $x:=R_{K}(\nu)$ (and the corresponding value is $h_{K}(\nu)$ ). The map

$$
R_{K} \mid \begin{aligned}
\mathbb{S}^{n-1} & \longrightarrow \partial K \\
\nu & \longmapsto x \text { such that } x \cdot \nu=\max _{y \in K} y \cdot \nu
\end{aligned}
$$

so defined is surjective; it is a bijection if and only $K$ is smooth. 
Proposition 1 Let $K \subset \mathbb{R}^{n}$ be a convex body of constant width $\alpha$. Then the following properties hold:

1. the diameter of $K$ is $\alpha$;

2. $K$ is strictly convex;

3. $K=\bigcap_{x \in \partial K} \overline{\mathrm{B}}(x, \alpha)$, where $\overline{\mathrm{B}}(x, \alpha)$ is the closed ball of center $x$ and radius $\alpha$;

4. if $K$ has a $\mathrm{C}^{2}$ boundary, then the radii of curvature at any point $x \in \partial K$ are all smaller than $\alpha$.

The proof of the proposition follows easily from a more technical lemma expressing how "farthest" points on $\partial K$ are related to the reverse Gauss map:

Lemma 1 Let $K \in \mathcal{W}_{\alpha}$; for any $x \in \partial K$, consider $F_{x}$ the set of points in $K$ which are as far as possible from $x$ :

$$
F_{x}:=\left\{x^{\prime} \in K ;\left|x-x^{\prime}\right|=\max _{y \in K}|x-y|\right\} .
$$

Then for any $x^{\prime} \in F_{x}$, we have $\left|x-x^{\prime}\right|=\alpha$ and $x=R_{K}\left(\frac{1}{\alpha}\left(x-x^{\prime}\right)\right)$.

This expresses the fact that if $x$ and $x^{\prime}$ are as far as possible, then they are at distance $\alpha$ and the unit vectors colinear to $x-x^{\prime}$ are support vector at $x$ or $x^{\prime}$.

Proof of the lemma and the proposition. Let $x \in \partial K$ be given. The set $F_{x}$ is nonempty since $K$ is compact. Let $x^{\prime} \in F_{x}$, and $\delta:=\left|x-x^{\prime}\right|=$ $\max _{y \in K}|x-y|$.

We have $\delta \leq \alpha$ since otherwise the projection of $K$ on the line joining $x$ and $x^{\prime}$ would have a length at least $\delta>\alpha$. So the diameter of $K$ is smaller than $\alpha$.

Let us show that $K$ is strictly convex. Assume by contradiction that there exists $y \neq x$ such that the segment $[x, y]$ is contained on $\partial K$. Let us denote by $x_{t}:=t x+(1-t) y$, with $t \in(0,1)$, the intermediate points in the segment. If $H$ is any support hyperplane at some $x_{t}$, it contains the whole segment. And given an outward unit normal vector $\nu$ to $H$, we have $x_{t} \cdot \nu=h_{K}(\nu)$ for all $t \in(0,1)$. Using again the compactness of $K$, there exists some $z \in \partial K$ such that $z \cdot(-\nu)=h_{K}(-\nu)$. Now $K$ has constant width, so

$$
\alpha=w_{K}(\nu)=x_{t} \cdot \nu-z \cdot \nu \leq\left|x_{t}-z\right| .
$$


The latter inequality must be an equality, since $K$ has diameter smaller than $\alpha$. This implies $x_{t}-z=\alpha \nu$, and this is not possible for all $t \in(0,1)$ since $x \neq y$.

Hence we have proved that $K$ is strictly convex. Repeating the same argument with $x$ instead of $x_{t}$, and $H$ any support hyperplane at $x$, we deduce again $x-z=\alpha \nu$. This implies $\delta=\alpha$, and also $z \in F_{x}$. Thus $K \subset \overline{\mathrm{B}}(x, \alpha)$; since this property holds for any $x \in \partial K$, we deduce that $K \subset \bigcap_{x \in \partial K} \overline{\mathrm{B}}(x, \alpha)$. Also if $K$ is $\mathrm{C}^{2}$ near $z$, this implies that the curvature radii at this point are smaller than $\alpha$.

Now for any given $x^{\prime} \in F_{x}$, let us define $\nu:=\frac{1}{\alpha}\left(x-x^{\prime}\right)$. Since $K \subset$ $\overline{\mathrm{B}}\left(x^{\prime}, \alpha\right)$, and since $\nu$ is the outward unit normal vector at $x$ on this ball, the hyperplane containing $x$ and directed by $\nu$ is a support plane to $K$. This implies $x=R_{K}(\nu)$ from the definition of the reverse Gauss map.

We have finally to prove $K \supset \bigcap_{x \in \partial K} \overline{\mathrm{B}}(x, \alpha)$, since the reverse inclusion was proved herebefore. Assume by contradiction that there exists some $z \in$ $\bigcap_{x \in \partial K} \overline{\mathrm{B}}(x, \alpha)$, such that $z \notin K$. Since $K$ is convex, it follows from the Hahn-Banach theorem that there exists $\nu \in \mathbb{S}^{n-1}$ such that $z \cdot \nu<x \cdot \nu$ for all $x \in K$. Consider $x:=R_{K}(\nu)$ and $x^{\prime}:=R_{K}(-\nu)$. From the previous study $x-x^{\prime}=\alpha \nu$ so $x \cdot \nu=\alpha+x^{\prime} \cdot \nu>\alpha+z \cdot \nu$. This contradicts $z \in \overline{\mathrm{B}}(x, \alpha)$.

For strictly convex bodies, we also have the following classical property:

Lemma 2 Let $K$ be a strictly convex body. Its support function $h_{K}$ is a $\mathrm{C}^{1}$ function on $\mathbb{S}^{n-1}$. It reverse Gauss map $R_{K}$ is continuous.

Proof. Let $\left(\nu_{i}\right) \subset \mathbb{S}^{n-1}$ be any converging sequence, with limit $\nu$. Define $x_{i}:=R_{K}\left(\nu_{i}\right)$. Since $K$ is compact, we may extract a subsequence (with no change of notation) in order to ensure that $\left(x_{i}\right)$ converges. Let $x$ be its limit. For all $y \in K$, and all $i$, we have $y \cdot \nu_{i} \leq x_{i} \cdot \nu_{i}$ from the definition of $R_{K}$. Passing to the limit yields $y \cdot \nu \leq x \cdot \nu$ for all $y \in K$, so $x=R_{K}(\nu)$ since this is the only maximizer of $y \mapsto y \cdot \nu$ from the strict convexity of $K$. This proves that $R_{K}$ is continuous.

Note that this implies that $h_{K}$ is continuous since $h_{K}(\nu)=\nu \cdot R_{K}(\nu)$. It is well-known that $h_{K}$ can be extended to a convex 1-homogeneous function $\bar{h}_{K}: \mathbb{R}^{n} \rightarrow \mathbb{R}$, also called the support function of $K$, and defined by $\bar{h}_{K}(d)=$ $|d| h_{K}\left(\frac{d}{|d|}\right)$. The subdifferential of $\bar{h}_{K}(d)$ at some $d \neq 0$ is the face of $K$ associated with $d$, that is $\left\{x \in K ; x \cdot d=\bar{h}_{K}(d)\right\}$ [3, Section D.3.1]. If $d=\nu \in \mathbb{S}^{n-1}$ and $K$ is strictly convex, this reduces to $\left\{R_{K}(\nu)\right\}$. Hence $\bar{h}_{K}$ is $\mathrm{C}^{1}$ on $\mathbb{S}^{n-1}$, and so is $h_{K}$. 


\section{Characterizations of bodies of constant width}

As explained before, finding bodies of constant width is not so easy. One simple way to construct a spheroform for instance, is to start with an orbiform having an axis of symetry, and then to consider the body of revolution generated by its rotation around the axis. However this process usually yields bodies with large volume.

We describe in the next section a new process that allows us to construct a body of constant width in dimension $n \geq 2$ from any body of constant width in dimension $n-1$. In order to do that, we need reciprocal to the properties given in the previous section, and in particular we need some characterizations of bodies of constant width.

Proposition 2 A strictly convex body $K$ has constant width $\alpha$ if and only if its reverse Gauss map satisfies:

$$
\forall \nu \in \mathbb{S}^{n-1}, \quad R_{K}(-\nu)=R_{K}(\nu)-\alpha \nu .
$$

Proof. If $K$ has constant width, we have from the definition of $R_{K}$ and taking into account the fact that the diameter of $K$ is smaller than $\alpha$ :

$$
\alpha=h_{K}(\nu)+h_{K}(-\nu)=\left(R_{K}(\nu)-R_{K}(-\nu)\right) \cdot \nu \leq\left|R_{K}(\nu)-R_{K}(-\nu)\right| \leq \alpha .
$$

Therefore we have equality in the above inequality, and this implies (6).

Let us assume that $K$ is strictly convex and satisfies (6). Then

$$
w_{K}(\nu)=h_{K}(\nu)+h_{K}(-\nu)=\left(R_{K}(\nu)-R_{K}(-\nu)\right) \cdot \nu=\alpha .
$$

So $K$ has constant width $\alpha$.

Let us draw a number of consequences of Proposition 2. Here and in the rest of the paper, a singular point $x$ on the boundary of some convex $K$ is a point where more than one unit outward support vector exists.

Corollary 1 Let $x$ be a singular point on the boundary of some $K \in \mathcal{W}_{\alpha}$. Then there exists a nontrivial arc of circle of radius $\alpha$ with center $x$ on $\partial K$.

A circle denotes as usual the intersection of some ball with a plane (dimension 2), and the center is in this plane. By "nontrivial" we mean that the arc must have more than one point.

Proof. The corollary follows from the proposition by noticing that for a convex set $K$, a point $x \in \partial K$ is singular if and only if $R_{K}^{(-1)}(x)$ contains more than one vector. By convexity it contains a spherical arc $\widehat{\nu}_{0} \nu_{1}$. From the proposition, $\partial K$ contains $x-\alpha \nu$ for all $\nu \in \widehat{\nu_{0} \nu_{1}}$, which is an arc of circle of radius $\alpha$. 
Corollary 2 In dimension $n \geq 3$, no finite intersection of balls have constant width, unless it reduces to a single ball.

Proof. Indeed consider $K=\bigcap_{i=1}^{m} \overline{\mathrm{B}}\left(x_{i}, r_{i}\right)$. We assume that this intersection is reduced, that is, no smaller intersection among the same balls yields the same set $K$. In particular, for each $i$, there is a relatively open part $Q_{i}$ of the boundary of $\overline{\mathrm{B}}\left(x_{i}, r_{i}\right)$ which is contained in $\partial K$. Any point $x \in Q_{i}$ is a differentiability point for $\partial K$, and $x=R_{K}(\nu)$ where $\nu=\left(x-x_{i}\right) / r_{i}$ is the common outward unit normal at $x$ to $\overline{\mathrm{B}}\left(x_{i}, r_{i}\right)$ and to $K$. If we denote by $\Sigma_{i} \subset \mathbb{S}^{n-1}$ the corresponding subset of unit vectors, we have $Q_{i}=R_{K}\left(\Sigma_{i}\right)$. According to Proposition 2, $R_{K}(\nu)-\alpha \nu \in \partial K$ for all $\nu$, in particular $\nu \in \Sigma_{i}$. This implies $r_{i} \leq \alpha$, for otherwise the corresponding image $\nu \mapsto R_{K}(\nu)-\alpha \nu$ yields a concave surface on $\partial K$, which is impossible.

So $r_{i} \leq \alpha$, and in particular, any nontrivial arc of circle of radius $\alpha$ on $\partial K$ has its center at some $x_{i}$. So the family of allowed centers for arcs of circle of radius $\alpha$ on $\partial K$ is finite. From the previous corollary, we deduce that $\partial K$ has only a finite number of singular points. This is clearly not possible in dimension $n \geq 3$, unless $\partial K$ is just a sphere.

Theorem 3 Let $K$ be a convex body. Then $K$ has constant width $\alpha$ if and only if it satisfies both conditions:

$$
\begin{gathered}
\operatorname{diam} K \leq \alpha \\
\forall x \in \partial K, \quad \exists x^{\prime} \in K,\left|x-x^{\prime}\right|=\alpha .
\end{gathered}
$$

Proof. We already know from Lemma 1 that a body with constant width satisfies these properties, so we just have to prove the reciprocal. Moreover the property is obvious in dimension $n=1$, so we assume that $n \geq 2$ in the following.

So assume that $K$ satisfies (7) and (8). Let us first prove that $K$ is strictly convex. Indeed if $\partial K$ contains a segment $\left[x_{0}, x_{1}\right]$ with nonempty (relative) interior, choose any $x$ in this interior, say $x=\left(x_{0}+x_{1}\right) / 2$. From condition (8), there exists $x^{\prime} \in K$ such that $\left|x-x^{\prime}\right|=\alpha$. This implies that $\left|x_{i}-x^{\prime}\right|>\alpha$ for $i=0$ or $i=1$, since a ball is strictly convex. So we get a contradiction with condition (7).

Now that we know that $K$ is strictly convex, we can use its reverse Gauss map and Proposition 2. Observe first that the strict convexity implies in particular that $K$ is $n$-dimensional (not contained in a strict affine subspace).

Let $\nu \in \mathbb{S}^{n-1}$ be given, and $x:=R_{K}(\nu)$. Let us first assume that $K$ is smooth at $x$, so that $\nu$ is the outward unit normal vector at $x$. From (8), there exists $x^{\prime} \in K$ such that $\left|x-x^{\prime}\right|=\alpha$. (Actually $x^{\prime} \in \partial K$ since $\operatorname{diam} K \leq \alpha$.) 
Since the ball $\overline{\mathrm{B}}\left(x^{\prime}, \alpha\right)$ contains $K$ from (7), the tangent hyperplanes to the ball and to $K$ at $x$ coincide. Therefore $\nu$ is equal to the unit outward normal vector to the ball $\overline{\mathrm{B}}\left(x^{\prime}, \alpha\right)$ at $x$, which is $\left(x-x^{\prime}\right) / \alpha$. Hence $x^{\prime}=x-\alpha \nu$, and in particular, for all $y \in K$, we have with (7):

$$
y \cdot(-\nu)=(x-y) \cdot \nu-x \cdot \nu \leq \alpha-x \cdot \nu=x^{\prime} \cdot(-\nu) .
$$

Taking the supremum on all $y$ yields $x^{\prime}=R_{K}(-\nu)$. So we have proved (6) for any $\nu$ such that $\partial K$ is smooth at $R_{K}(\nu)$. Let us recall that the subset of points where the boundary is smooth is dense in $\partial K$.

So consider an arbitrary $\nu, x:=R_{K}(\nu)$ and $x^{\prime}:=R_{K}(-\nu)$. Notice that $x^{\prime} \neq x$ since $K$ is $n$-dimensional. The sets $U:=R_{K}^{(-1)}(x)$ and $V:=-R_{K}^{(-1)}\left(x^{\prime}\right)$ are closed in the sphere $\mathbb{S}^{n-1}$ and have a common element, namely $\nu$. However none of them contains $-\nu\left(\right.$ since $\left.x^{\prime} \neq x\right)$, so they are not equal to the whole sphere. Since it is not possible to have each one included in the interior of the other, one of $\partial U \cap V$ or $U \cap \partial V$ must be nonempty (the boundary here is considered with respect to the sphere $\left.\mathbb{S}^{n-1}\right)$. Say for instance $\partial U \cap V \neq \emptyset$, so that there exists $\nu^{\prime} \in \partial U \cap V$. In particular we can find a sequence $\left(\nu_{k}\right)_{k \geq 1}$ converging to $\nu^{\prime}$ as $k \rightarrow \infty$ such that $x_{k}:=R_{K}\left(\nu_{k}\right) \neq x$ for all $k$. (Note that $R_{K}$ is continuous according to Lemma 2 , so $x_{k}$ converges to $x$.) We may even assume, with no loss of generality, that $\partial K$ is smooth at $x_{k}$ for all $k \geq 1$, since the set of such points is dense.

In particular $x_{k}-\alpha \nu_{k}=R_{K}\left(-\nu_{k}\right)$ from our previous study. Letting $k$ going to infinity yields $x-\alpha \nu^{\prime}=R_{K}\left(-\nu^{\prime}\right)$. But the latter is $x^{\prime}$ since we assumed $\nu^{\prime} \in V=-R_{K}^{(-1)}\left(x^{\prime}\right)$. So we have proved $\nu^{\prime}=\nu_{0}$ where $\nu_{0}:=$ $\left(x-x^{\prime}\right) / \alpha$. This shows that $\partial U \cap V=\left\{\nu_{0}\right\}$ whenever this set is nonempty. In general, we have $\partial U \cap V \subset\left\{\nu_{0}\right\}$. A symetrical argument shows that $U \cap \partial V \subset\left\{\nu_{0}\right\}$ also. This means that one of the two sets $U$ or $V$ has empty interior. In particular, since $\nu \in U \cap V$, we get $\nu=\nu_{0}$. This proves (6).

Later on we will need a slightly different version of this theorem:

Theorem 4 Let $K$ be a closed subset of $\mathbb{R}^{n}$. Then $K$ is a convex body of constant width $\alpha$ if and only if it satisfies (7) and

$$
\forall x \in \partial K, \exists x^{\prime},\left[x, x^{\prime}\right] \subset K \text { and }\left|x-x^{\prime}\right|=\alpha .
$$

The difference is that we do not assume $K$ convex here, but require instead that the whole segment $\left[x, x^{\prime}\right]$ is contained in $K$.

Proof. It is clear that (9) implies (8). So we just have to prove that $K$ is convex, and use Theorem 3 to conclude the proof.

So assume by contradiction that $K$ is not convex. Hence there exists $x_{0}, x_{1} \in K$ such that $\left[x_{0}, x_{1}\right] \not \subset K$. Let $x$ be some point in $\left[x_{0}, x_{1}\right] \cap \partial K$. 
We can find $x^{\prime} \in K$ with $\left|x-x^{\prime}\right|=\alpha$ using (9). Since $x \in\left[x_{0}, x_{1}\right]$, we have $\left|x-x_{0}\right|+\left|x-x_{1}\right|=\left|x_{1}-x_{0}\right|$. From Ptolemy's inequality we get, taking into account that $\operatorname{diam} K \leq \alpha$ :

$$
\begin{aligned}
\alpha\left|x_{1}-x_{0}\right| & \leq\left|x-x_{0}\right|\left|x_{1}-x^{\prime}\right|+\left|x-x_{1}\right|\left|x_{0}-x^{\prime}\right| \\
& \leq \alpha\left(\left|x-x_{0}\right|+\left|x-x_{1}\right|\right)=\alpha\left|x_{1}-x_{0}\right| .
\end{aligned}
$$

So there must be equality everywhere, which means that the four points are cocyclic, and that $\left|x_{i}-x^{\prime}\right|=\alpha$ for $i=0,1$. Note that this implies in particular that the four points are not aligned. Therefore the assumption $x \in\left[x_{0}, x_{1}\right] \cap \partial K$ implies $x=x_{0}$ or $x=x_{1}$.

So we proved that for any $(x, y) \in K^{2}$, the whole interior of the segment $[x, y]$ is outside $K$ or is interior to $K$. In particular $x_{0} \in \partial K$, so there exists $x_{0}^{\prime}$ such that $\left|x_{0}-x_{0}^{\prime}\right|=\alpha$ and $\left[x_{0}, x_{0}^{\prime}\right] \subset K$. More precisely the interior of this segment is included in the interior of $K$. In particular, for any $x \in\left(x_{0}, x_{0}^{\prime}\right)$, we have $\left[x, x_{1}\right] \subset K$ since $x \notin \partial K$. This implies that $\left[x_{0}, x_{1}\right] \subset \partial K$ by passing to the limit $x \rightarrow x_{0}$, a contradiction.

The preceding characterizations are useful, but the diameter condition is difficult to handle in the variationnal context we consider in [4]. So let us give a slightly different characterization of bodies of constant width.

Theorem 5 Let $K$ be closed subset of $\mathbb{R}^{n}$. Then $K$ has constant width $\alpha$ if and only if it satisfies:

$\forall \nu \in \mathbb{S}^{n-1}, \exists x_{\nu} \in K$

$$
x_{\nu}+\alpha \nu \in K \quad \text { and } \quad \forall y \in K,\left(y-x_{\nu}\right) \cdot \nu \in[0, \alpha] .
$$

So (10) expresses that the projection of $K$ on the line $\mathbb{R} \nu$ is included in an interval of length $\alpha$ (condition $\forall y \ldots$ ) and that the corresponding extremal points $x_{\nu}$ and $x_{\nu}^{\prime}:=x_{\nu}+\alpha \nu$ do exist in $K$.

Proof. If $K \in \mathcal{W}_{\alpha}$, it satisfies (10) with $x_{\nu}=R_{K}(-\nu)$. Indeed we know from Proposition 2 that $x_{\nu}+\alpha \nu=R_{K}(\nu)$ in that case, and the remaining part follows from the very definition of $R_{K}$.

So let us prove the converse, starting with some closed set satisfying (10). Let us first prove that $\operatorname{diam} K \leq \alpha$. Let $x, y \in K$ be given, with $x \neq y$, and consider $\nu:=(y-x) /|y-x|$. From (10), there exists $x_{\nu} \in K$ such that $\left(y-x_{\nu}\right) \cdot \nu$ and $\left(x-x_{\nu}\right) \cdot \nu$ both belong to $[0, \alpha]$. Since

$$
\left(y-x_{\nu}\right) \cdot \nu=|y-x|+\left(x-x_{\nu}\right) \cdot \nu
$$


according to the definition of $\nu$, this implies in particular that $|y-x| \leq \alpha$. So diam $K \leq \alpha$.

Let $\hat{K}$ be the closed convex hull of $K$. Notice that $\hat{K}$ also satisfies (10) since $K \subset \hat{K}$ and $y \in \hat{K}$ implies that $y$ is a finite convex combination of elements of $K$. In particular, $\hat{K}$ has diameter $\leq \alpha$, too.

Consider some $x \in \partial \hat{K}$. Since $\hat{K}$ is convex, there exists some outward support vector $\nu \in \mathbb{S}^{n-1}$. So we have $x \cdot \nu \geq y \cdot \nu$ for all $y \in \hat{K}$. Let $x_{\nu}$ be given by (10), and $x_{\nu}^{\prime}:=x_{\nu}+\alpha \nu \in K$. Since $\left(x-x_{\nu}^{\prime}\right) \cdot \nu \leq 0$ from (10) and $\left(x-x_{\nu}^{\prime}\right) \cdot \nu \geq 0$ from the definition of $\nu$, we deduce that $\left(x-x_{\nu}^{\prime}\right) \cdot \nu=0$. Therefore

$$
\left|x-x_{\nu}\right|^{2}=\left|x-x_{\nu}^{\prime}\right|^{2}+\left|x_{\nu}-x_{\nu}^{\prime}\right|^{2}=\left|x-x_{\nu}^{\prime}\right|^{2}+\alpha^{2} .
$$

Since this is also less than $\alpha^{2}$ from the diameter property, we have proved that $x=x_{\nu}^{\prime}$. Therefore the point $x^{\prime}:=x_{\nu}$ satisfies $\left|x-x^{\prime}\right|=\alpha$ and $x^{\prime} \in \hat{K}$.

We have proved that $\hat{K}$ satisfies (7) and (9), so $\hat{K} \in \mathcal{W}_{\alpha}$ according to Theorem 4. In particular, $\hat{K}$ is strictly convex. Therefore any $x \in \partial \hat{K}$ is exposed. Since such an $x$ can be expressed as a convex combination of $(n+1)$ points of $K$ according to Caratheodory's Theorem [7, Theorem 1.1.4], and since the exposure property implies that all these points coincide with $x$, we deduce that $x \in K$. Hence $K=\hat{K}$ and this concludes the proof.

\section{Raising dimensions}

Now we have the required elements to exhibit the raising dimensions process:

Theorem 6 Let $H \subset \mathbb{R}^{n}$ be an affine hyperplane, $E_{+}$and $E_{-}$the two open half-spaces separated by $H$, and $K_{0} \subset H$ be an $(n-1)$-dimensional body of constant width $\alpha$. Let $Q$ be any set satisfying

$$
K_{0} \subset Q \subset \bar{E}_{-} \cap \bigcap_{x \in K_{0}} \overline{\mathrm{B}}(x, \alpha)
$$

Consider the set $K$ defined as follows:

$$
\begin{aligned}
& K \cap \bar{E}_{+}=K_{+}:=\bar{E}_{+} \cap \bigcap_{x \in Q} \overline{\mathrm{B}}(x, \alpha) \\
& K \cap E_{-}=K_{-}:=E_{-} \cap \bigcap_{x \in K_{+}} \overline{\mathrm{B}}(x, \alpha) .
\end{aligned}
$$

Then $K$ is a $n$-dimensional body of constant width $\alpha$, and $K \cap H=K_{0}$. 

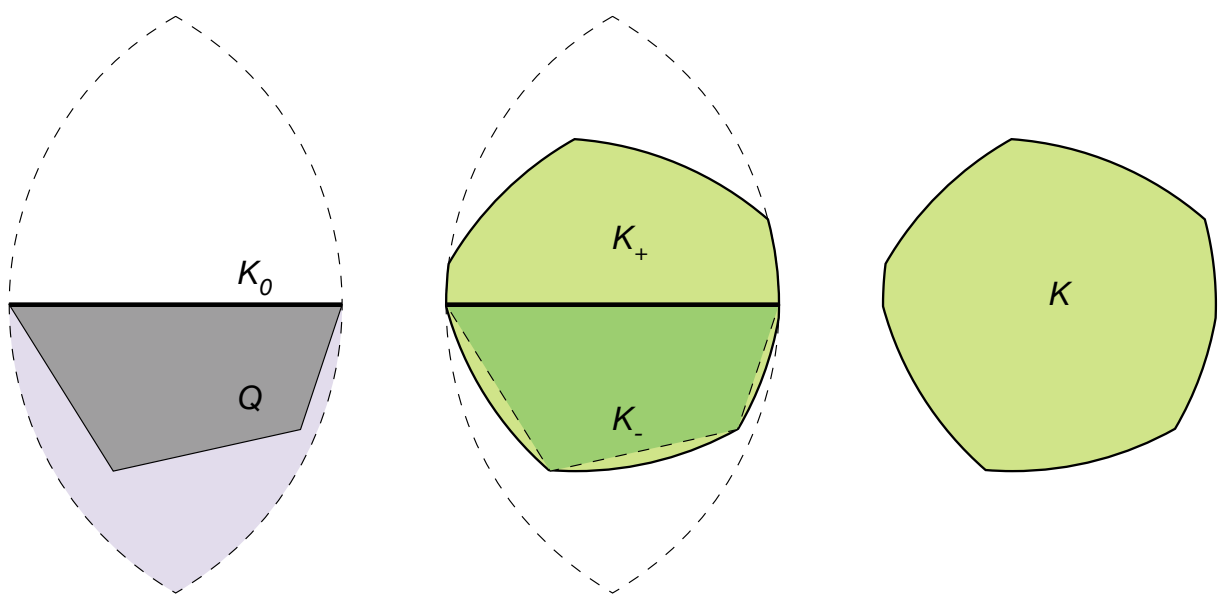

Figure 1: Illustration of the raising dimension process, 1D to 2D.

The property $K_{0}=K \cap H$ shows that this process raises dimension. Note that since $K_{0} \subset H$ and has diameter $\alpha$, it is possible to find sets $Q$ satisfying (11).

The simplest choice for $Q$ is just $Q=K_{0}$. With this choice, and starting from a one-dimensional convex body (a segment of width $\alpha$ ), we get a twodimensional Reuleaux triangle. Starting from a two-dimensional disk, we get a rotated Reuleaux triangle. Starting from a two-dimensional Reuleaux triangle, we get Meissner's body.

Let us explain that in more details. The Figure 1 shows an example that makes the construction of the Theorem 6 easier to understand. We start from a 1-dimensional convex body of constant width, that is a segment $K_{0}$ of length $\alpha$ (bold on the left and middle parts of the figure). In the figure, $H$ is the horizontal line, $E_{+}$and $E_{-}$are the upper and lower part of the plane. All the construction takes place in the intersection of balls of radius $\alpha$ on $K$, which reduces here to the intersection of two balls (dashed lines). We choose a set $Q$ satisfying (11), that is in the lower part of this intersection (shown in light gray). In our example, $Q$ is a quadrilateral (gray on the left of the figure). The set $K_{+}$is the intersection of balls centered on $Q$ (only four balls really since $Q$ is a polygon) and the upper part of the plane (middle part of the figure). Then $K_{-}$is the intersection of balls centered on $K_{+}$(again a finite number of balls is enough here), and it contains $Q$ (dashed lines). The resulting set $K$ is their union, and is shown again on the right. It is now easier to understand that if we had chosen $Q=K_{0}$, then $K_{+}$would have been the upper part in the intersection of the two disks, and then $K_{-}$would 

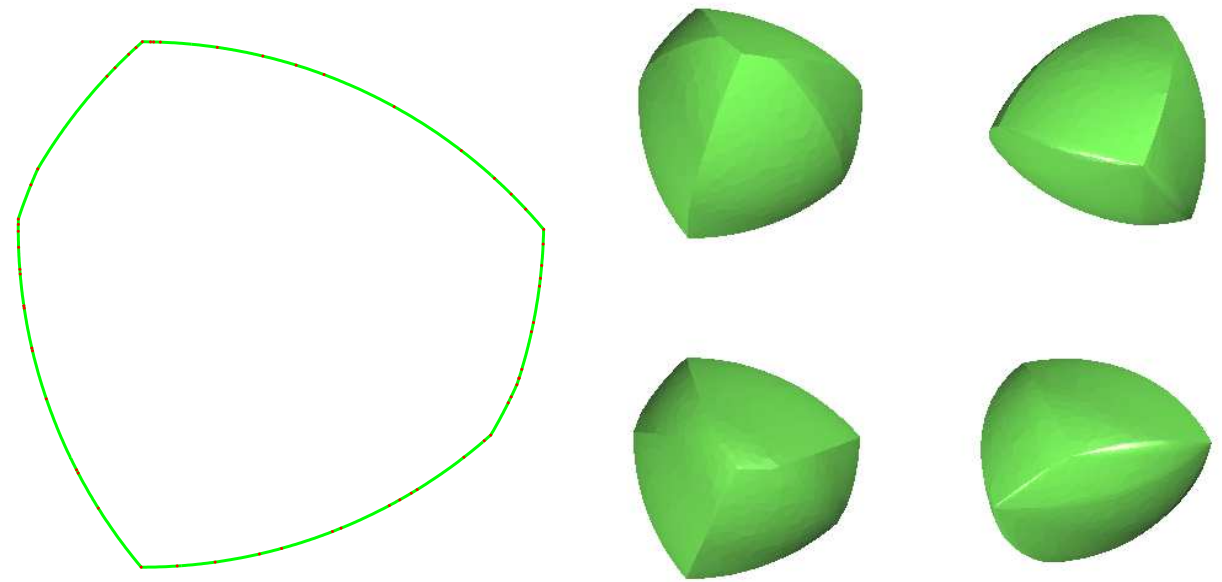

Figure 2: Illustration of the raising dimension process, 2D to 3D.

have been the intersection of the disk centered on the upper point of $K_{+}$with the lower half plane. So we would get a Reuleaux triangle as claimed.

The Figure 2 shows an example starting from a two-dimensional body $K_{0}$ of constant width shown on the left. This body is the intersection of a rather large number of disks. We chose $Q=K_{0}$ in the construction. The resulting three-dimensional body is shown on the right (with a different scale). Notice in particular on the lower left part, the body is shown from above, so the projection is just $K_{0}$.

Should we have started from a Reuleaux triangle $K_{0}$, then $K_{+}$would have been the intersection of the upper-space and the three balls centered on vertices of $K_{0}$. Then $K_{-}$would have been determined from $K_{+}$as defined in the theorem, but would have not been a finite intersection of disks, according to Corollary 2. The resulting spheroform has an upper part that is identical to a spherical tetrahedron: that is exactly one of the variant of Meissner's body, shown in Figure 3. (The detailed construction of this body is given in [2].)

There is no limit on the dimensions that can be reached by the process given by Theorem 6. Starting from Meissner's body $K_{0}$, and using $Q=K_{0}$, we get a four dimensional body shown in Figure 4. (The fourth dimension is shown by different parallel cross-sections.)

For the proof of the Theorem, we need a small geometrical lemma: 

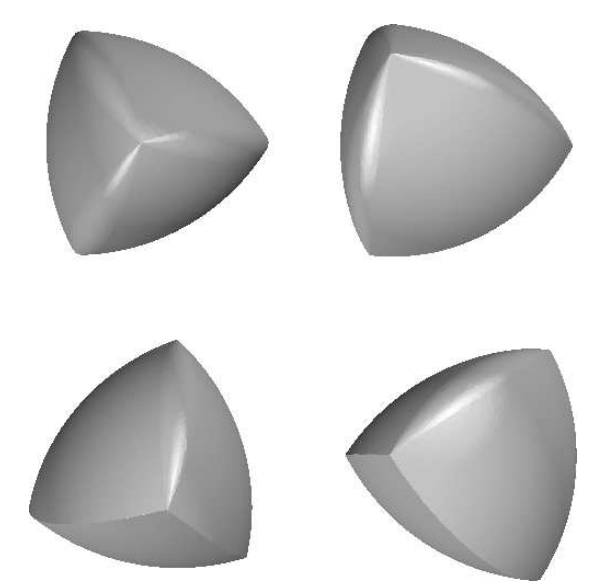

Figure 3: One of the two forms of Meissner's body. We can get this using Theorem 6 with $K_{0}=Q$ a Reuleaux triangle.

Lemma 3 Let $a, b, x, y$ be four points in a plane. Assume that

$$
\max (|a-x|,|a-y|,|b-x|,|b-y|) \leq|b-a|
$$

and that the segment $[x, y]$ does not intersect the line generated by a and $b$. Then $|x-y| \leq|b-a|$.

Proof. Let $\alpha:=|b-a|$ and $c$ be the point at distance $\alpha$ from $a$ and $b$, on the same side of the line generated by $a$ and $b$ than $x$ and $y$. So $a, b, c$ forms an equilateral triangle. Let $T$ be the Reuleaux triangle supported by this triangle. From the assumptions on $x, y$, we see that these two points belong to $T$. Since $T$ has constant width $\alpha$, its diameter is $\alpha$ also from Lemma 1 .

Proof of Theorem 6. Observe first that $K_{+}$is closed and $K_{+} \cap H=K_{0}$ from its definition and (11). Also $\bar{K}_{-} \cap H=K_{0}$, and $K_{-} \cap K_{+}=\emptyset$. The set $K_{-}$is not closed, but $\bar{K}_{-}=K_{-} \cup K_{0}$. So in particular $K=K_{+} \cup K_{-}$is closed, and $K \cap H=K_{0}$ as claimed.

We will prove that $K$ is convex and satisfies both conditions of Theorem 3 . (Note that it is obvious that $K_{+}$and $K_{-}$are convex, but it is not so clear that $K=K_{+} \cup K_{-}$is.)

Let $x, y$ be any two different points in $K$, and define $\nu:=(y-x) /|y-x| \in$ $\mathbb{S}^{n-1}$. We will prove that $[x, y] \subset K$ and $|x-y| \leq \alpha$. Note that we don't need to prove that for any pair $(x, y)$ in order to prove that $K$ is convex and has diameter $\alpha$. It is enough to consider a dense subset, since $K$ is closed, 

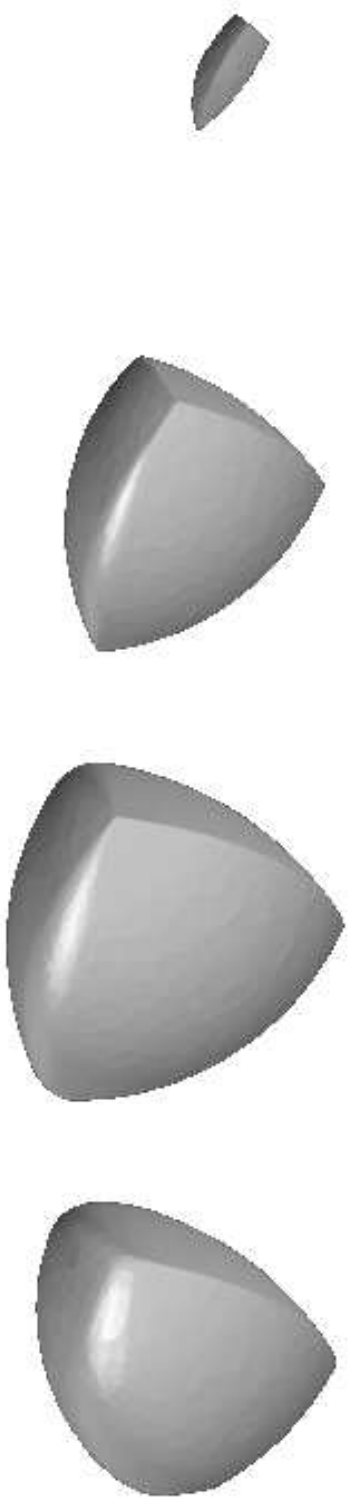
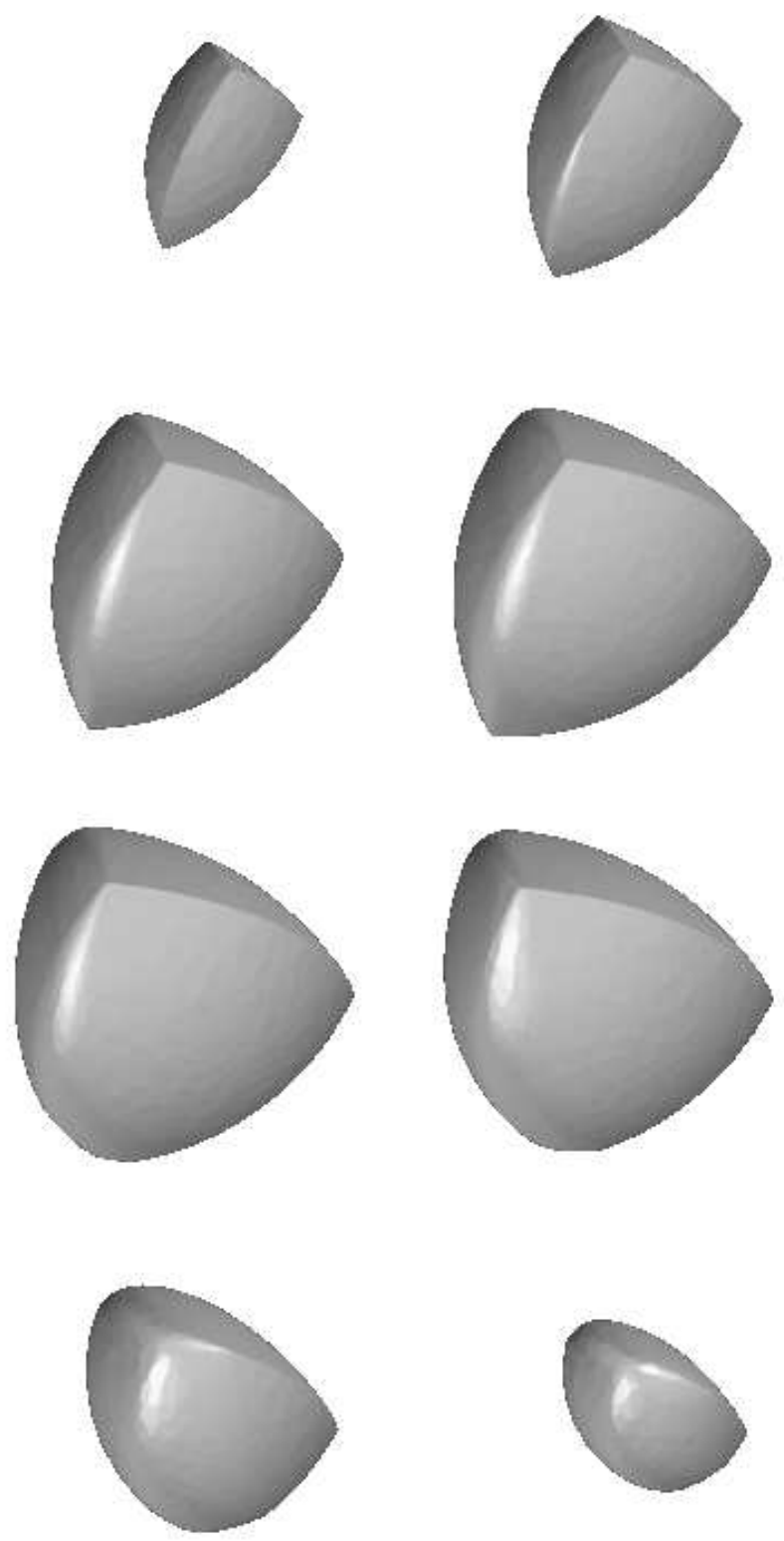

Figure 4: A four dimensional body of constant width (parallel cross-sections), obtained using Theorem 6 with $K_{0}=Q$ a Meissner's body. 
so we may assume that the straight line $\delta$ joining $x$ and $y$ (that is $x+\mathbb{R} \nu$ ) is not parallel to $H$, and that $\delta \cap H \not \subset \partial K_{0}$.

From these assumptions, $\delta$ intersects $H$ at a point $z \notin \partial K_{0}$. Let $p \in \partial K_{0}$ be defined as follows:

1. if $z \notin K_{0}$, let $p$ be the orthogonal projection of $z$ onto $K_{0}$ (in $H$ );

2. otherwise $z$ is in the relative interior of $K_{0}$; let $p$ be the farthest point from $z$ in $K_{0}$ (so that $|p-z| \geq|m-z|$ for all $m \in K$ ).

We define also $\nu_{0} \in \mathbb{S}^{n-1}$ by $\nu_{0}:=(z-p) /|p-z|$ in the first case, and $\nu_{0}:=(p-z) /|p-z|$ otherwise. Note that this vector belongs to the vector space directing $H$. We claim that $p=R_{K_{0}}\left(\nu_{0}\right)$ in all cases. Indeed let us just check the two different cases in the same order:

1. if $p \neq p_{0}:=R_{K_{0}}\left(\nu_{0}\right)$, then $p \cdot \nu_{0}<p_{0} \cdot \nu_{0}$. Since $z-p \in \mathbb{R}_{+}^{*} \nu_{0}$ by definition, this implies $0<\left(p_{0}-p\right) \cdot(z-p)$ in contradiction to the fact that $p$ is the projection of $z$ onto the convex set $K_{0}$;

2. again if $p \neq p_{0}:=R_{K_{0}}\left(\nu_{0}\right)$, then $p \cdot \nu_{0}<p_{0} \cdot \nu_{0}$. Since $p-z \in \mathbb{R}_{+}^{*} \nu_{0}$ by definition we get

$$
0>2\left(p_{0}-p\right) \cdot(z-p)=|z-p|^{2}-\left|z-p_{0}\right|^{2}+\left|p-p_{0}\right|^{2} .
$$

Hence we have $\left|z-p_{0}\right|>|z-p|$ in contradiction to the fact that $p$ is the farthest point from $z$ in $K_{0}$.

Now let us consider $p^{\prime}:=p-\alpha \nu_{0}$. We know from Lemma 1 that $p^{\prime}=$ $R_{K_{0}}\left(-\nu_{0}\right) \in \partial K_{0}$ since $K_{0}$ has constant width.

Let $P$ be the two dimensional plane $p+\mathbb{R} \nu+\mathbb{R} \nu_{0}$. It contains $z$, and also the four points $x, y, p, p^{\prime}$. So in particular these four points are coplanar.

We now discuss the different cases:

- if $x \in K_{+}$and $y \in K_{+}$, then $[x, y] \subset K_{+}$since $K_{+}$is convex, and we have from its definition that $K_{+} \subset \overline{\mathrm{B}}(p, \alpha) \cap \overline{\mathrm{B}}\left(p^{\prime}, \alpha\right)$. In particular, we get:

$$
|x-p| \leq \alpha,\left|x-p^{\prime}\right| \leq \alpha,|y-p| \leq \alpha,\left|y-p^{\prime}\right| \leq \alpha .
$$

Since $\left|p-p^{\prime}\right|=\alpha$, we get $|x-y| \leq \alpha$ using Lemma 3 (with $a=p$, $\left.b=p^{\prime}\right)$.

- if $x \in K_{-}$and $y \in K_{-}$, we get the same results using similar arguments (note that $K_{0} \subset K_{+}$, so (14) holds true again). 
- if one of the point is in $K_{+}$, say $x$, and the other in $K_{-}$, we get $|x-y| \leq$ $\alpha$ from the definition of $K_{-}$. Here $x$ and $y$ are on opposite sides of the hyperplane $H$, so in particular $z$ lies on the segment $[x, y]$, that is $z=[x, y] \cap H$. Remember also that $z$ is contained in the straight line generated by $p$ and $p^{\prime}$. Since $K_{+}$and $K_{-}$are contained in $\overline{\mathrm{B}}(p, \alpha) \cap$ $\overline{\mathrm{B}}\left(p^{\prime}, \alpha\right)$ by definition, $z$ lies on the projection of $\overline{\mathrm{B}}(p, \alpha) \cap \overline{\mathrm{B}}\left(p^{\prime}, \alpha\right)$ onto the straight line generated by $p$ and $p^{\prime}$. But such a projection is just the segment $\left[p, p^{\prime}\right]$, so we have $z \in\left[p, p^{\prime}\right] \subset K_{0}$. In particular $z \in K_{+}$, so $[x, z] \subset K_{+}$, and $z \in \bar{K}_{-}$, so $[z, y] \subset \bar{K}_{-}$. This implies $[x, y] \subset K$.

So we proved that $K$ is convex and $\operatorname{diam} K \leq \alpha$. To conclude the proof we need to prove (8). So let us consider some $x \in \partial K$. Note that the property is obvious if $x \in K_{0}$, since $K_{0}$ has constant width and $K_{0}=K \cap H$.

If $x \in \partial K_{-} \backslash K_{0}$, let us define $\delta:=\sup _{y \in K_{+}}|x-y|$. Note that $\delta \leq \alpha$ from the definition of $K_{-}$, and that there exists $x^{\prime} \in K_{+}$such that $\left|x-x^{\prime}\right|=\delta$ since $K_{+}$is compact. So if $\delta=\alpha,(8)$ is satisfied. If on the contrary $\delta<\alpha$, then there exists a (small) open neighborhood $U$ of $x$ such that $U \subset \overline{\mathrm{B}}(y, \alpha)$ for all $y \in K_{+}$. Since $x \in E_{-}$, an open set, we may assume that $U \subset E_{-}$, reducing the neighborhood if necessary. So $U \subset K_{-}$, in contradiction to the assumption $x \in \partial K_{-}$.

If $x \in \partial K_{+} \backslash K_{0}$, let us define similarly $\delta:=\sup _{y \in \bar{Q}}|x-y|$. Again there is some $x^{\prime} \in \bar{Q}$ such that $\left|x-x^{\prime}\right|=\delta$ since $\bar{Q}$ is compact. If $\delta<\alpha$ there is some open neighborhood $U$ of $x$ such that $U \subset E_{+}$and $U \subset \overline{\mathrm{B}}(y, \alpha)$ for all $y \in \bar{Q}$. So we get also a contradiction. Hence $\delta=\alpha$ and $x^{\prime} \in \partial Q$. This latter relation implies in particular $x^{\prime} \in K$. Indeed, from the definition of $K_{+}$we have

$$
\forall y \in \bar{Q}, \forall z \in K_{+}, \quad|y-z| \leq \alpha .
$$

Since $Q \subset \bar{E}_{-}$from (11), this implies $\bar{Q} \subset \bar{K}_{-} \subset K$.

\section{References}

[1] T. Bonnesen, W. Fenchel, Theory of convex bodies, BCS Associates, 1987, p. 135-149.

[2] G. D. Chakerian, H. Groemer, Convexity and its Applications, ed P. M. Gruber and J. M. Wills, Birkhauser, 1983, p. 49-96.

[3] J. B. Hiriart-Urruty, C. Lemaréchal, Fundamentals of convex analysis, Springer-Verlag, 2001.

[4] T. LAChAnd-Robert \& E. Oudet, to appear. 
[5] F. Meissner,

[6] R. T. Rockafellar, Convex Analysis, Princeton University Press (1970).

[7] R. Schneider, Convex bodies: the Brunn-Minkowski theory, Cambridge Univ. Press, 1993. 